\title{
Virtualization-Based Digitization of a Retail Store: An Enhanced Implementation of Digital Transformation
}

\author{
B.K Praveen Kumar, K. Santhi Sree
}

\begin{abstract}
The rise of neoteric technologies like Machine Learning, the Internet of Things, Cloud Services, etc has affected the life of a common man at various levels. Irrespective of the size or domain, almost all companies are now incorporating digitization to various degrees and thus progressing towards a new business model with little or no significance to geographical and physical barriers. This shift from traditional store models to automated entities is referred to as Digital Transformation. It is the simplified way of outlining how digital technologies are transforming and automating business operations across all organizations irrespective of their domain. This digital revolution relies on a whole range of machinery, networks, services, and operations to expand their power of communication, thus ensuring seamless integration with the technologies. At this juncture, there would be many challenges in both technical and non-technical aspects. To facilitate successful automation by resolving those issues, using the concept of virtualization can be very helpful. Virtualization is the process of creating a virtual instance of hardware resources like virtual applications, servers, or storage by logically separating them from the hardware. It enables multiple applications or operations to gain access to the hardware resources/ software resources of the host machine. In a sense, virtualization is always at the center of all this revolution providing a rock-solid foundation. For example, when digitizing an organization, machine learning algorithms are applied to the IoT data in addition to the organizational data. Given the huge size of data, companies adapting to this automation rely on cloud services for data management because of the reliability it provides. This issue is solved using the Edge Computing concept which is an advanced implementation of Virtualization. In this paper, we try to discuss such challenges and try to understand how virtualization can be useful in solving them. This can be exemplified using a hypothetical digitalization in a retail store scenario.
\end{abstract}

Keywords: Digital Transformation, IoT, Cloud Services, Machine Learning, Edge Computing, Virtualization...

Manuscript received on September 07, 2021.

Revised Manuscript received on September 30, 2021.

Manuscript published on September 30, 2021

* Correspondence Author

B.K Praveen Kumar*, Department of Data Science Specialization School of Information Technology, Jawaharlal Nehru Technological University, Hyderabad India. bommanakondapraveenkumar@gmail.com

Dr. K. Santhi Sree, Department of Computer Science and Engineering, School of Information Technology, Jawaharlal Nehru Technological University (JNTUH), Hyderabad, India. E-mail: kakara_2006@jntuh.ac.in

(c) The Authors. Published by Blue Eyes Intelligence Engineering and Sciences Publication (BEIESP). This is an open access article under the CC BY-NC-ND license (http://creativecommons.org/licenses/by-nc-nd/4.0/)

\section{INTRODUCTION}

The advent of digital and social media platforms has led to a meteoric rise in data and changed the way people communicate with each other. This along with the evolution of various digital technologies like Artificial Intelligence, Advanced Analytics, Internet of Things, Augmented and Virtual Reality, etc., is pushing various organizations to adopt these technologies by transforming their key business operations and thus enabling Digital Transformation (DX) in organizations across various domains. This transformation has the power to progressively branch out and boost productivity across all sectors and industries. Digital Revolution is about advancing the organization by implementing new technologies in various realms of the organization. Thus fast-paced revolution is now diminishing the barriers between the physical and virtual worlds by inventing new digital business models.

\section{DIGITAL TRANSFORMATION}

\section{A. Goals}

Since the digitally enabled business models are comparatively recent, there are no standardized definitions or procedures on how to adapt to this transformation. However, an organization is said to be digitally transformed if it succeeds in implementing new technologies to perform one or more of the following activities:

1. Launch a digital model of the organization:

- Every organization is expected to have a digital twin in the form of a website and an application.

- Having a website or an online shopping site not only brings the organization closer to the clients but also makes the collection of data easier.

2. Implement privacy providing technologies:

- Every organization should gain the trust of endusers to be successful. Data security is one of the factors that affect the trust level of end-users especially in a digital model which collects data at every other step.

- Thus, implementing technologies like blockchain to secure the data might reinforce the trust of clients and users

Published By:

Blue Eyes Intelligence Engineering and Sciences Publication (BEIESP)

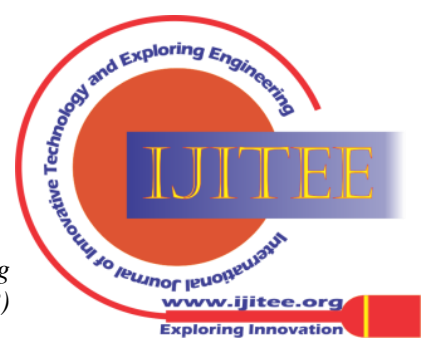


3. Management of the end-product digitally:

- Every organization should start viewing its end project as a product and should be able to manage them digitally.

- This allows automation and independence of units from internal and external factors. Technologies like Data Analytics, Cloud, etc., can help in achieving this goal.

4. Augmented Intelligence:

- Augmented intelligence is an alternative conceptualization of artificial intelligence that focuses on AI's assistive role in advancing human capabilities.

- Using AI-Powered Assistants holds the potential to regenerate automate routine labor and amplify work.

Digital transformation is not about automating the existing business processes but integrating every phase of your organization with suitable technologies. Everything traditional must be reconsidered including the ideas and management decisions. An organization is said to be digitally successful if it can integrate your business process with a digital platform that can create personalized experiences for the customers by collecting data, handle customer queries and enable social media marketing on a large scale.

The digital transformation can be complex to adapt to in the beginning but confers great results in the long term. Smart policies which can minimize the cost are to be adopted by the organization. A smart strategy should be able to create an effective framework to follow throughout the process. Digitalization will further diverge into every walk of life and ignoring it will only lead to the loss of the organization that fails to adopt new technologies.

Despite the resistance of people and their personal opinions, digitalization should be accommodated. The perfect plan of action consists of all the factors and policies that adapt according to the situations driven by digitalization. This framework should also be based on the following factors:

$>$ Past experiences and processes

$>$ People of the organization and their culture

$>$ Changes in management and communication

$>$ New Innovations and Leadership

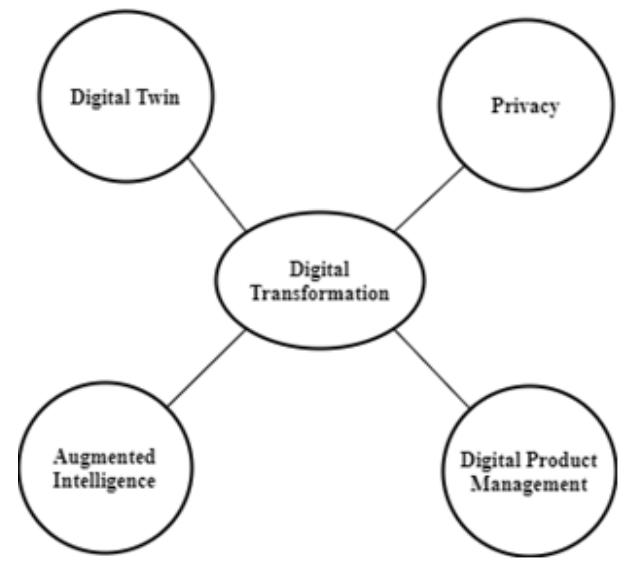

Fig-1: Digital Transformation

\section{B. Challenges:}

\section{RETAIL STORE SIMULATION}

In the modern-day, shopping malls have become an integral part of the city culture and have become more of entertainment rather than an essential activity. New malls are expanding their horizons every day. Because of the competition, unless a shopping center has unique features, it can incur losses. Thus, retail stores are also transforming digitally and becoming "SMART".

Retail stores are deploying smart technologies to create digital connections that benefit both parties. With the internet at the heart of the interaction, providing free Wi-Fi is no longer optional for malls that want to attract shoppers and keep them engaged. Moreover, once the mobile device is connected to the store's network, it opens more doors to engage the customers than just Wi-Fi. By adapting to these technologies and utilizing the data from mobile devices, a shopper can receive personalized experiences from the vendor like music, customized recommendations, and offers. This approach has the potential to attract a lot more customers because of the comfort it provides and the time it saves.

To achieve this level of personalization, a shopping mall would have required some devices like beacons and geofences. The customers would have to download a specially designed app. These devices mine the data and then after analyzing the data they can send customized offers and personal recommendations. However, these physical devices can be limited in their capabilities. To achieve our specified goals of automating the shopping mall, these devices should be powered by some technologies. In this paper, a small example is shown of how a shopping environment can undergo digital transformation by employing various technologies like machine learning, edge computing, cloud services, and virtualization.

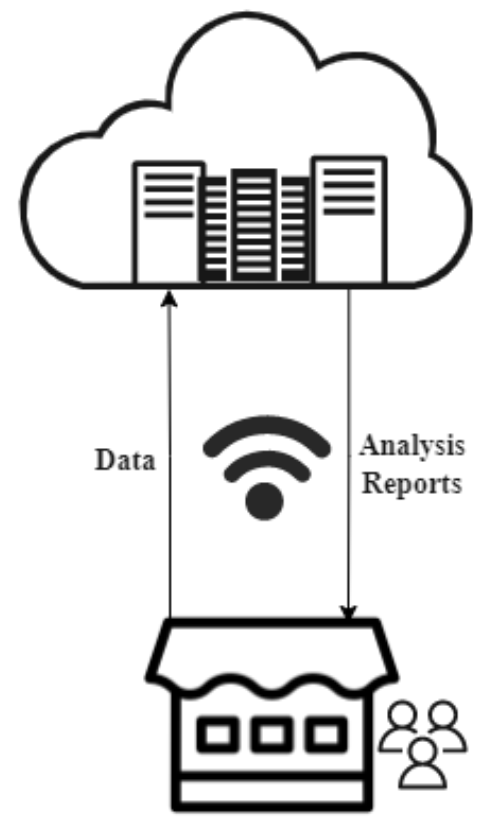

Fig-2: Traditional Retail Stores

Published By:

Blue Eyes Intelligence Engineering and Sciences Publication (BEIESP)

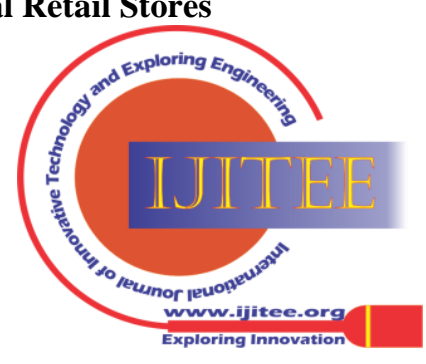




\section{PROPOSED MODEL}

To avoid communication delays, software upgrades and faster devices can be used but that would be costly and impractical. A better alternative is to store the most used in the cloud as usual. This process of caching the data at an edge node is referred to as edge computing. Here, we consider a Smart Retail Store and simulate the edge computing situation using Virtual Machines and Google Cloud. In this proposed model, we use Cloud to store different types of data and apply machine learning algorithms to the whole data. We then create virtual machines to store the frequently used data on the machine and then apply pre-trained machine learning models to these partial datasets. This includes the following modules:

1. Creation of Virtual Machines:

A virtual machine is created for each type of partial dataset to represent the on-site edge nodes. A pretrained deep learning model is stored here to predict outcomes.

\section{Request of Cloud Resources:}

The original data is all stored in the cloud and even machine learning algorithms are applied here to this data. Google Cloud is chosen because it is exceptionally reliable and secure.

3. Deep Learning Model:

As mentioned earlier, a pre-trained deep learning model for each type of dataset is stored in the virtual machine. We chose the deep learning model because it requires little customization and parameter tuning. Also, they offer portability and thus can be distributed across multiple machines.

Since we need to simulate a Smart Store, we use three different types of datasets.

$>$ Customer Reviews Dataset to recommend according to their choices and taste

> Fashion Dataset to predict perfect fitted clothes to the customer

> Music recommendation dataset to play music based on the emotions of the customer.

These datasets are downloaded from various sites like Kaggle for the Spotify dataset and Customer Review datasets.

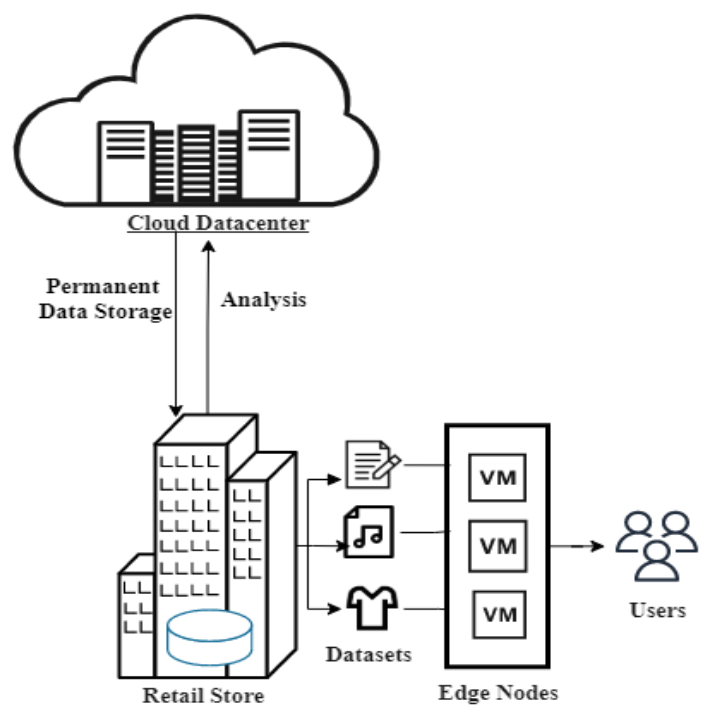

Fig-3: Proposed Model data nearer to the site and the rest of the data can be stored

\section{EXPERIMENT}

The whole datasets are stored in the Google cloud and the data is analyzed for valuable information and insights using Google Cloud Data Analytics. However, the required datasets are stored in the local virtual machines. The Reviews dataset is stored in one virtual machine, the sample music data is stored in another, and the fashion dataset is stored in yet another virtual machine locally. Each virtual machine consists of a cached machine learning model trained using the original dataset. Thus, the analysis of recent data is faster than the analysis of whole data and the personalized recommendations and suggestions can be given faster since they are closer to the end-user. The user accesses these recommendations through an application on the mobile.

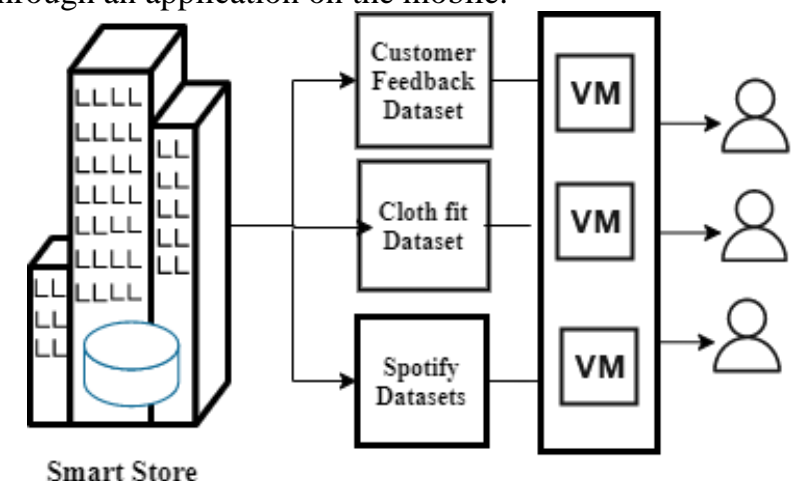

Fig-4: Block Diagram

\section{RESULTS}

We considered three dataset modules as recently used data. For the Customer Reviews Dataset, the output was a model with $90 \%$ of accuracy.

$\begin{array}{lr}5922 & \text { Negative } \\ 15017 & \text { Positive } \\ 2977 & \text { Positive } \\ 22761 & \text { Neutral } \\ 26482 & \text { Positive } \\ 7861 & \text { Positive } \\ 21027 & \text { Positive } \\ 16242 & \text { Positive } \\ 13791 & \text { Positive } \\ 6434 & \text { Positive } \\ 16813 & \text { Positive } \\ 22 & \text { Positive } \\ 26772 & \text { Positive } \\ 18775 & \text { Positive } \\ 648 & \text { Positive } \\ 15522 & \text { Positive }\end{array}$

Fig-5: Customer Reviews- Predictions

The music is first grouped into various moods and then according to our facial expression, the music is suggested. The accuracy of the music recommended by facial recognition can be given as a matrix.

Published By:

Blue Eyes Intelligence Engineering and Sciences Publication (BEIESP) 


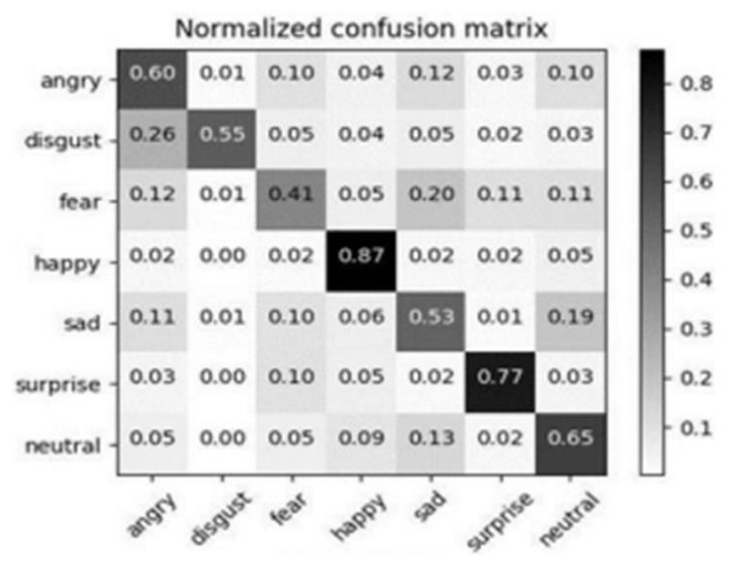

Fig-6: Music recommendation system

Using the cloth fit dataset, the size of the body is first calculated, and the fit is estimated according to the model. Only three body sizes are considered for ease of convenience. The model estimates the fit up to $80 \%$ accuracy. The accuracy of the cloth fit module is given by the following charts.

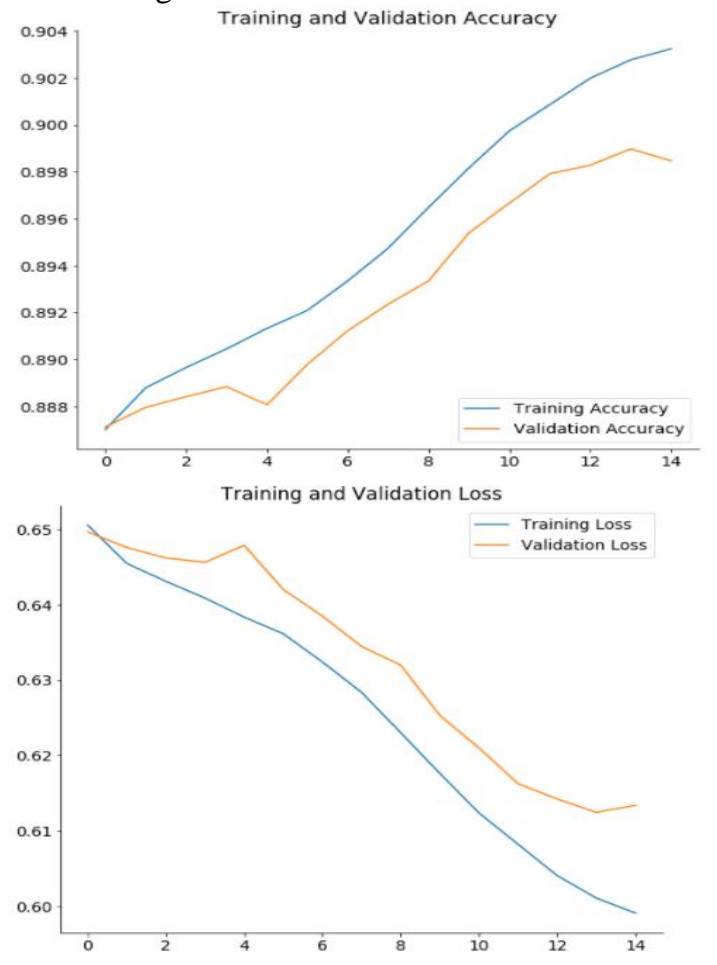

Fig-7: Cloth fit System Performance

These modules are timed and when compared to the original cloud service model give faster performance and higher reliability in a simulated environment.

\section{CONCLUSION}

This paper outlines various problems caused by the integration of technologies within a brick-and-mortar organization. It also explains a simple approach using the virtualization concept to solve the issues. The simulation was a restricted model, but the solution is more effective in real-time applications. It is more effective when combined with IoT data because of the huge size of data. Creating a project in the cloud gives us the flexibility to add resources and remove them as per requirement. Virtualization increases the security of the cloud by protecting both the integrity of guest virtual machines and cloud components.

Published By:

Blue Eyes Intelligence Engineering and Sciences Publication (BEIESP) 136 (C) Copyright: All rights reserved.
To improve this model, real-time IoT data can be used to provide suggestions.

\section{REFERENCES}

1. Nanda, Susanta \& Chiueh, tzi-cker. (2005). A Survey on Virtualization Technologies.

2. K. Chandrasekaran. 2014, Essentials of Cloud Computing(1st. ed.). Chapman \&amp; Hall/CRC.

3. Geeta, \& Prakash, Shiva. (2019). Role of Virtualization Techniques in Cloud Computing Environment. 10.1007/978-981-13-0344-9_37.

4. Mann, A. Virtualization 101: Technologies, benefits, and challenges. White Paper, EMA Boulder, CO.

5. Carbone, M., W. Lee, and D. Zamboni. Taming virtualization. IEEE Security and Privacy 6(1): 65-67, 2008.

6. VMware, "Vmware http://www.vmware.com/products/desktop/ws features.html.

7. Jay Munro, "Virtual machines and VMWare," PC Magazine, December 2001 [8]Xing Y., Zhan Y. (2012) Virtualization and Cloud Computing. In: Zhang Y. (eds) Future Wireless Networks and Information Systems. Lecture Notes in Electrical Engineering, vol 143. Springer, Berlin, Heidelberg.

8. https://doi.org/10.1007/978-3-642-27323-0_39

9. https://dzone.com/articles/virtual-clusterswith-virtual-machines

10. Tarek Abdelzaher, Yifan Hao, Kasthuri Jayarajah, Archan Misra, Per Skarin, Shuochao Yao, Dulanga Weerakoon,and Karl-Erik Årzén. 2020. Five Challenges in Cloud-enabled Intelligence and Control. ACM Trans.Internet Technol. 20, 1, Article 3 (February 2020), 19 pages.

11. https://whatfix.com/digitaltransformation/\#dt_industries

12. https://hbr.org/2020/05/digitaltransformation-comes-down-to-talentin-4key-areas

13. https://whatfix.com/resources/casestudy/how-emarketplace-takealotautomateself-training-for-their-sellers/

14. https://www.dta.gov.au/digitaltransformation-strategy/impactdigitalrevolution

15. https://www.mckinsey.com/businessfunctions/organization/ourinsights/theorganization-blog/getting-your-organizationready-for-adigital-transformation

16. https://www.itpro.co.uk/612016/what-isvirtualisation

\section{AUTHORS PROFILE}

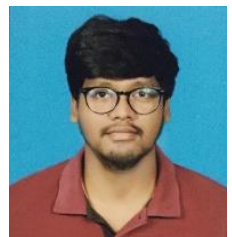

BK Praveen Kumar, is currently pursuing his Master of Technology degree in Data Science specialization from School of Information Technology, Jawaharlal Nehru Technological University, Hyderabad. He completed his Bachelor of Technology in Computer Science specialization at Guru Nanak Institute of Technology, Hyderabad. Equally passionate about coding, Web Development and Data Analytics, his areas of interest are Data Engineering, Deep Learning, Human Computer Interaction and Artificial Intelligence. He published a previous paper titled " Covid-19: A Tentative Estimation of Fatality Rates using Random Forest Algorithm" in the International Journal of Computer Applications under the esteemed guidance of Dr. K. Santhi Sree (Professor, School of Information Technology)

E-mail: bommanakondapraveenkumar@gmail.com.

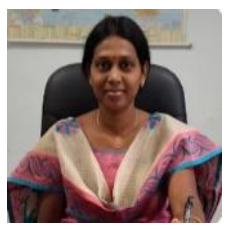

Dr. K. Santhi Sree (www.drksanthisree.com), Dr. K. Santhi Sree(www.drksanthisree.com), presently a Professor in the Department of Computer science and Engineering at School of Information Technology, Jawaharlal Nehru Technological University(JNTUH) in Hyderabad. She is a recipient of many awards like Mother Theresa Global Award (2017), Bharat Ratna Dr.A.P.J Abdul Kalam Excellence Award, Best Citizens of India Award (2015), Excellence in Education Award (April 2015), Bharat Jyothi Award (June 2015) etc.She is an editorial board member for many publications. She also serves as a Program Committee member, BOS member for many JNTU affiliated colleges of JNTUH. A Professional member of ISTE,CSI,IEI,IETE,IAENG,IACSIT. She has published more than 50 papers in reputed journals and has successfully guided more than 70 M.Tech projects. 